Supplement of Atmos. Chem. Phys., 16, 13081-13104, 2016

http://www.atmos-chem-phys.net/16/13081/2016/

doi:10.5194/acp-16-13081-2016-supplement

(C) Author(s) 2016. CC Attribution 3.0 License.

(c) (i)

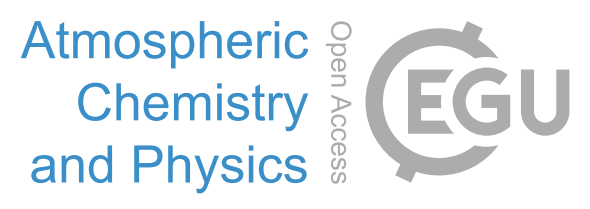

Supplement of

\title{
Impact of climate change on the production and transport of sea salt aerosol on European seas
}

\section{Joana Soares et al.}

Correspondence to: Joana Soares (joana.soares@fmi.fi)

The copyright of individual parts of the supplement might differ from the CC-BY 3.0 licence. 
$1 \frac{d F}{d D_{p}}=1.373 * U_{10 m}^{3.41} * \frac{1+0.057 D_{p}^{1.05}}{D_{p}^{3}} * 10^{1.19 \exp \left(-\left(\frac{0.38-\lg D_{p}}{0.65}\right)^{2}\right)}$

3 where $d F / d D_{p}$ is the rate of sea salt droplet generation per unit area of the whitecap and per

4 increment of droplet dry radius $\left(d D_{p}\right) \mathrm{U}_{10}$ is the wind speed at $10 \mathrm{~m}$

5

$6 \frac{d F}{d D_{p}}=3.84 * 10^{-6} * A_{k} T_{w}+B_{k} * U_{10 m}^{3.41}$

where $d F / d D_{p}$ is the rate of sea salt droplet generation per unit area of sea surface and per increment of $\log$ of the droplet dry radius $\left(d D_{p}\right), \mathrm{T}_{\mathrm{w}}$ is the temperature of seasurface water,

10 and $A_{k}$ and $B_{k}$ are the parameters describing the dependence of sea salt flux on the aerosol

11 size (described in Eq. S3), and $\mathrm{U}_{10}$ is the wind speed at $10 \mathrm{~m}$.

$$
A_{k}=C_{4} d_{d}^{4}+C_{3} d_{d}^{3}+C_{2} d_{d}^{2}+C_{1} d_{d}+C_{0}
$$

$$
B_{k}=D_{4} d_{d}^{4}+D_{3} d_{d}^{3}+D_{2} d_{d}^{2}+D_{1} d_{d}+D_{0}
$$

13 Where $C_{i}$ and $D_{i}$ are empirical coefficients tabulated according to Mårtensson et al. (2003), 14 shown in the table below.

16 Table S1: Coefficients for the Parameterization of $A_{k}\left(C_{4}-C_{0}\right)$ and $B_{k}\left(D_{4}-D_{0}\right)$ in Equation 17 (S3) for the Three Size Intervals (k)

\begin{tabular}{llllll}
\hline Size Interval, $\mathbf{1 0}^{-6} \mathbf{~ m}$ & $\mathbf{C}_{\mathbf{4}}$ & $\mathbf{C}_{\mathbf{3}}$ & $\mathbf{C}_{\mathbf{2}}$ & $\mathbf{C}_{\mathbf{1}}$ & $\mathbf{C}_{\mathbf{0}}$ \\
\hline $0.020-0.145$ & $-2.576 \times 10^{35}$ & $5.932 \times 10^{28}$ & $2.867 \times 10^{21}$ & $-3.003 \times 10^{13}$ & $2.881 \times 10^{6}$ \\
$0.145-0.419$ & $-2.452 \times 10^{33}$ & $2.404 \times 10^{27}$ & $-8.148 \times 10^{20}$ & $1.183 \times 10^{14}$ & $-6.743 \times 10^{6}$ \\
$0.419-2.8$ & $1.085 \times 10^{29}$ & $-9.841 \times 10^{23}$ & $3.132 \times 10^{18}$ & $-4.165 \times 10^{12}$ & $2.181 \times 10^{6}$ \\
\hline Size Interval, $\mathbf{1 0}^{-6} \mathbf{m}$ & $\mathrm{D}_{4}$ & $\mathrm{D}_{3}$ & $\mathrm{D}_{2}$ & $\mathrm{D}_{1}$ & $\mathrm{D}_{0}$ \\
\hline $0.020-0.145$ & $7.188 \times 10^{37}$ & $-1.616 \times 10^{31}$ & $6.791 \times 10^{23}$ & $1.829 \times 10^{16}$ & $7.609 \times 10^{8}$ \\
$0.145-0.419$ & $7.368 \times 10^{35}$ & $-7.310 \times 10^{29}$ & $2.528 \times 10^{23}$ & $-3.787 \times 10^{16}$ & $2.279 \times 10^{9}$ \\
$0.419-2.8$ & $-2.859 \times 10^{31}$ & $2.601 \times 10^{26}$ & $-8.297 \times 10^{20}$ & $1.105 \times 10^{15}$ & $-5.800 \times 10^{8}$ \\
\hline
\end{tabular}



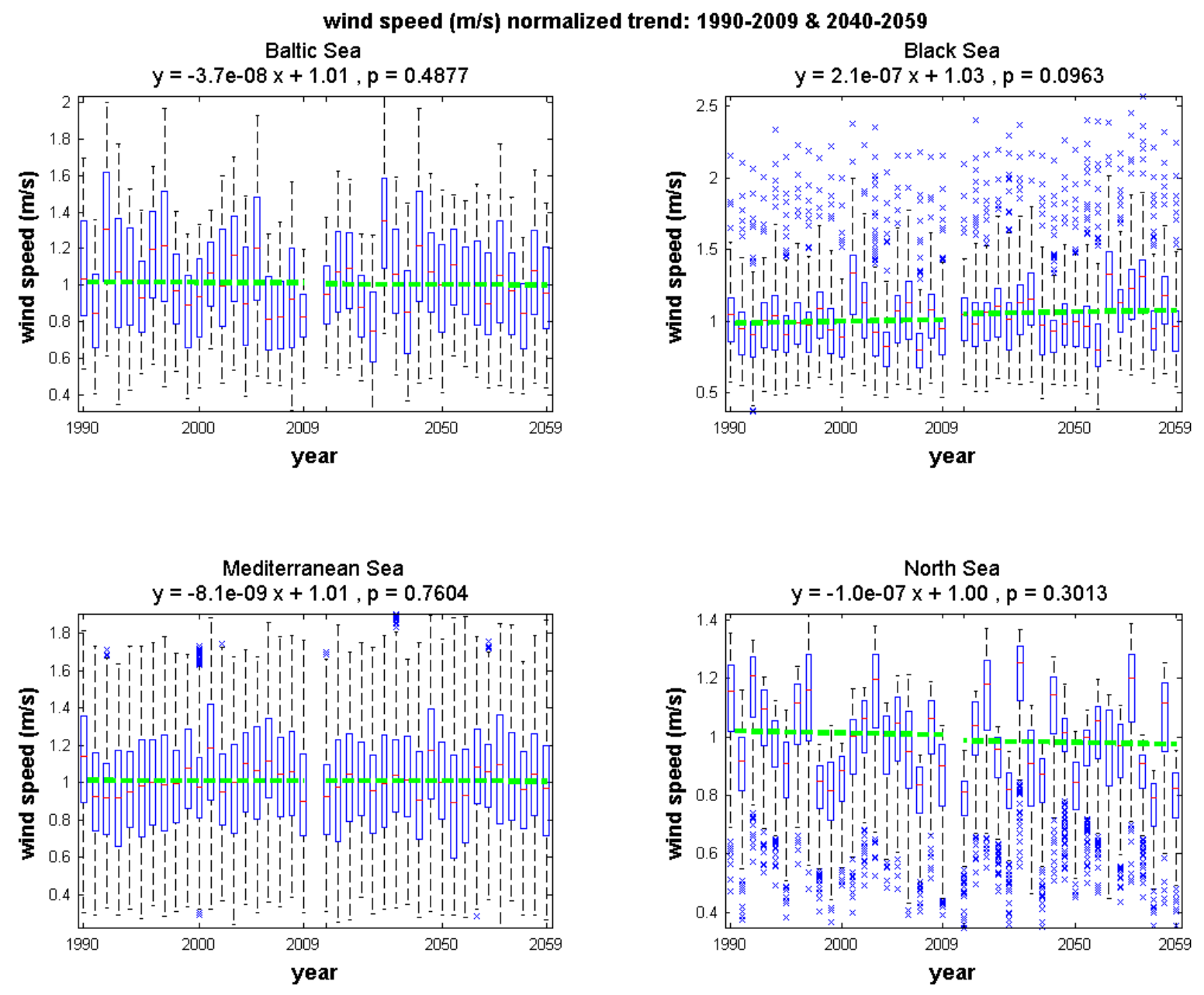

3 Figure S1 Annual mean win speed $\left(\mathrm{m} \mathrm{s}^{-1}\right)$ normalized trend $(\mathrm{y})$ over the past and future 4 periods (x [year]), over the Baltic, Black, North and Mediterranean Seas. Only sea cells 5 considered. 

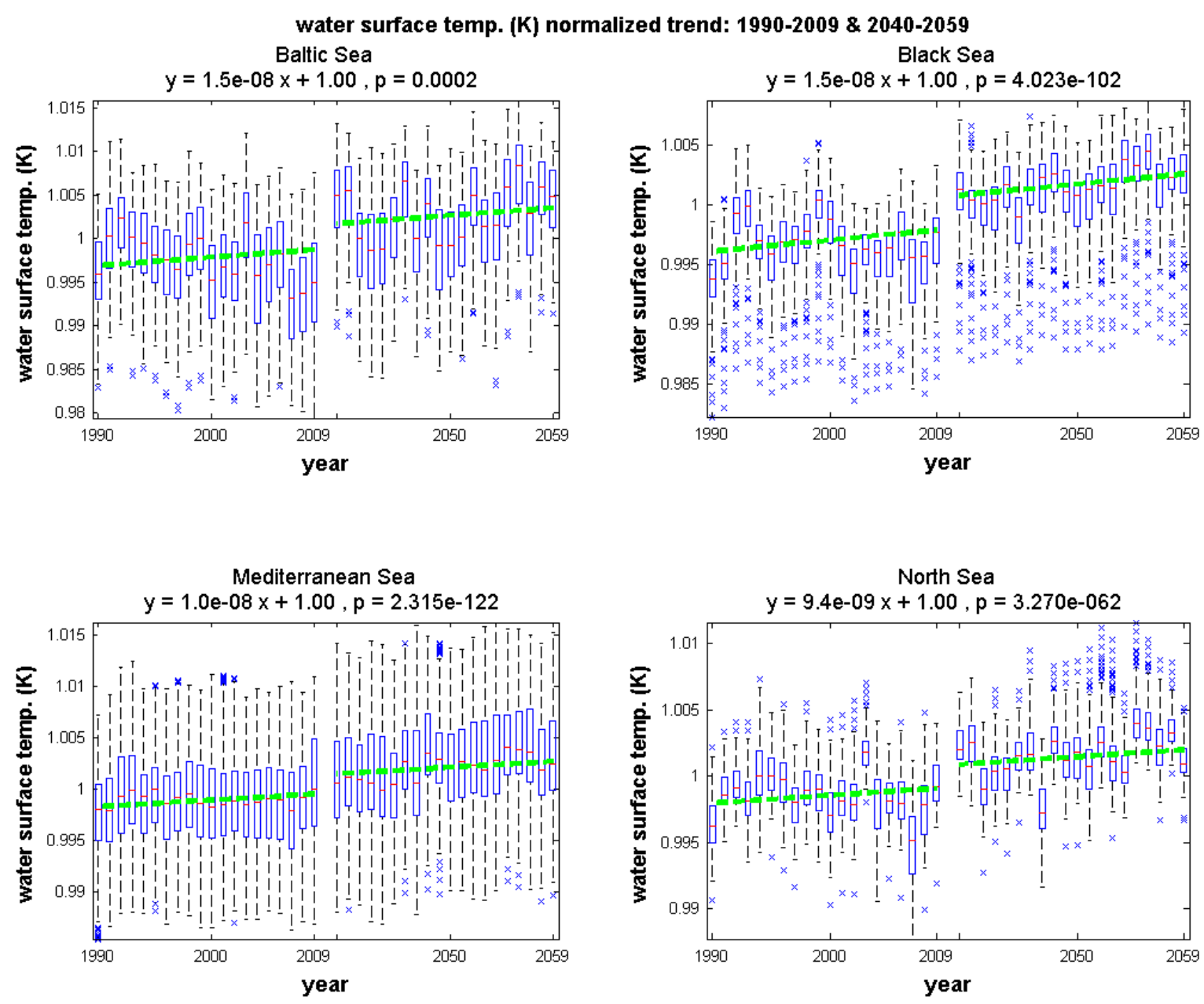

3 Figure S2 Annual mean water surface temperature $(\mathrm{K})$ normalized trend (y) over the past and

4 future periods (x [year]), over the Baltic, Black, North and Mediterranean Seas. Only sea cells 5 considered. 

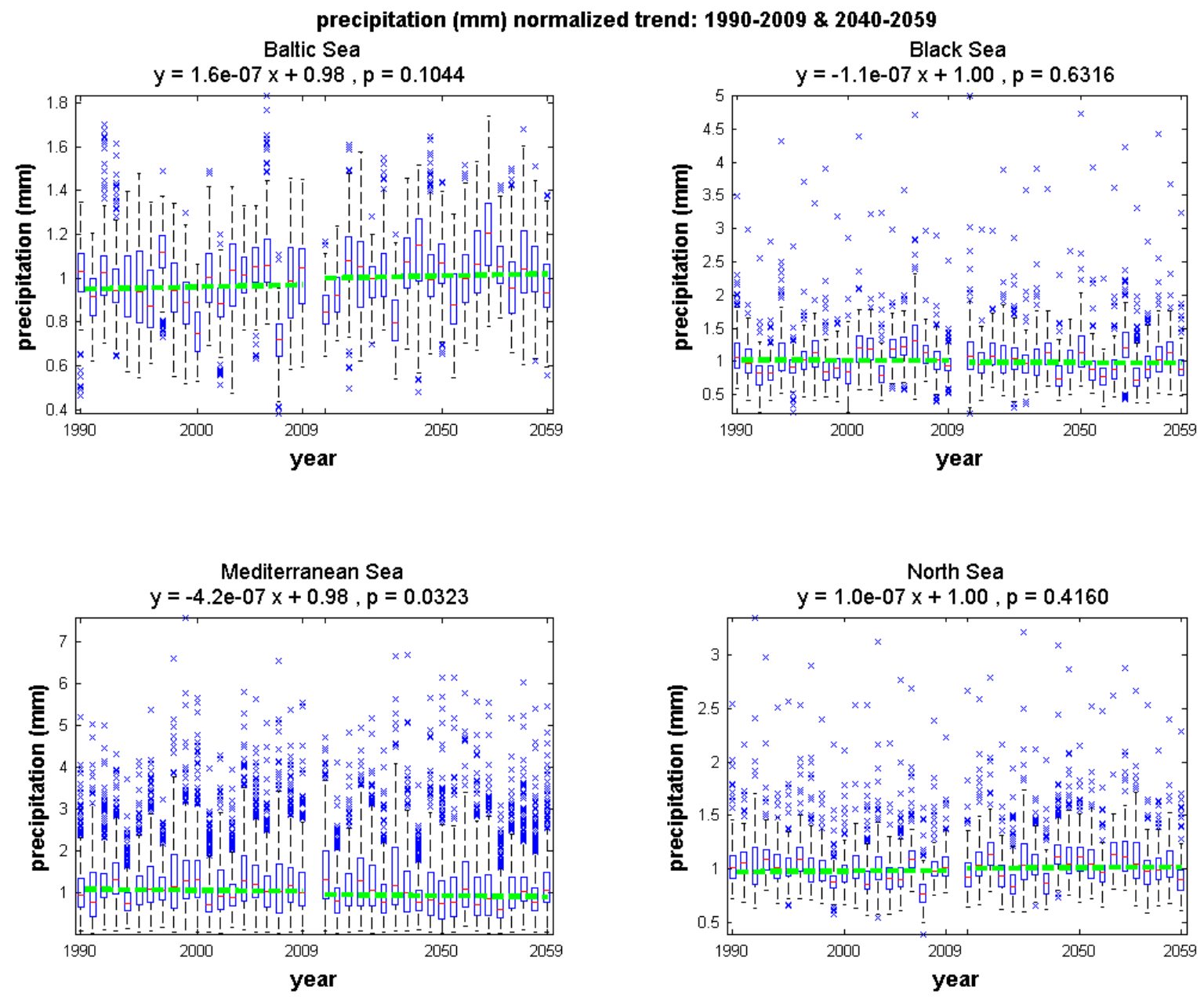

3 Figure S3 Annual precipitation ( $\mathrm{mm}$ ) normalized trend (y) over the past and future periods ( $\mathrm{x}$ 4 [year]), over the Baltic, Black, North and Mediterranean Seas. Only sea cells considered. 


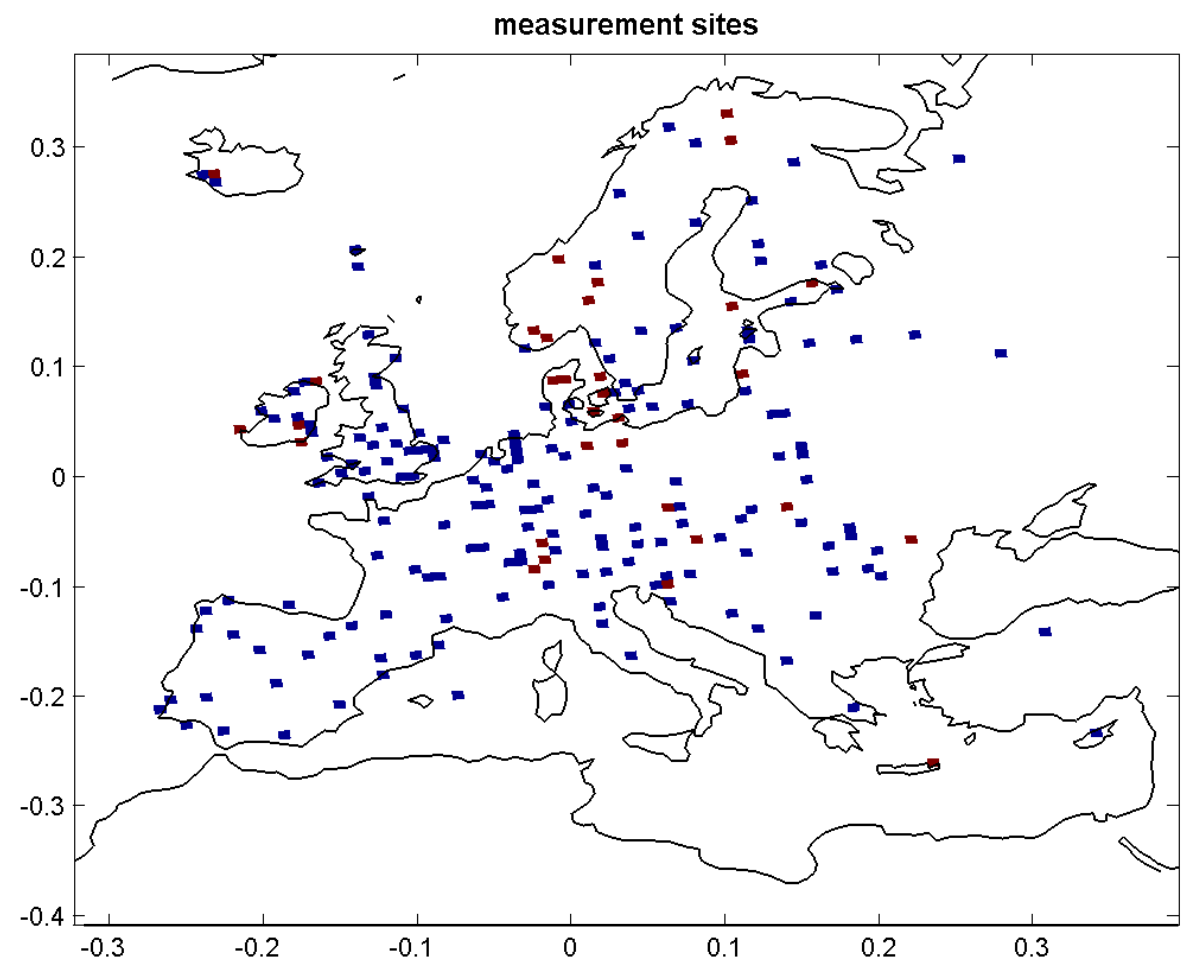

3 Figure S4 Location of the EMEP measurement sites measuring concentration and wet 4 deposition of $\mathrm{Na}^{+}$. The ones measuring both quantities are marked in red. 

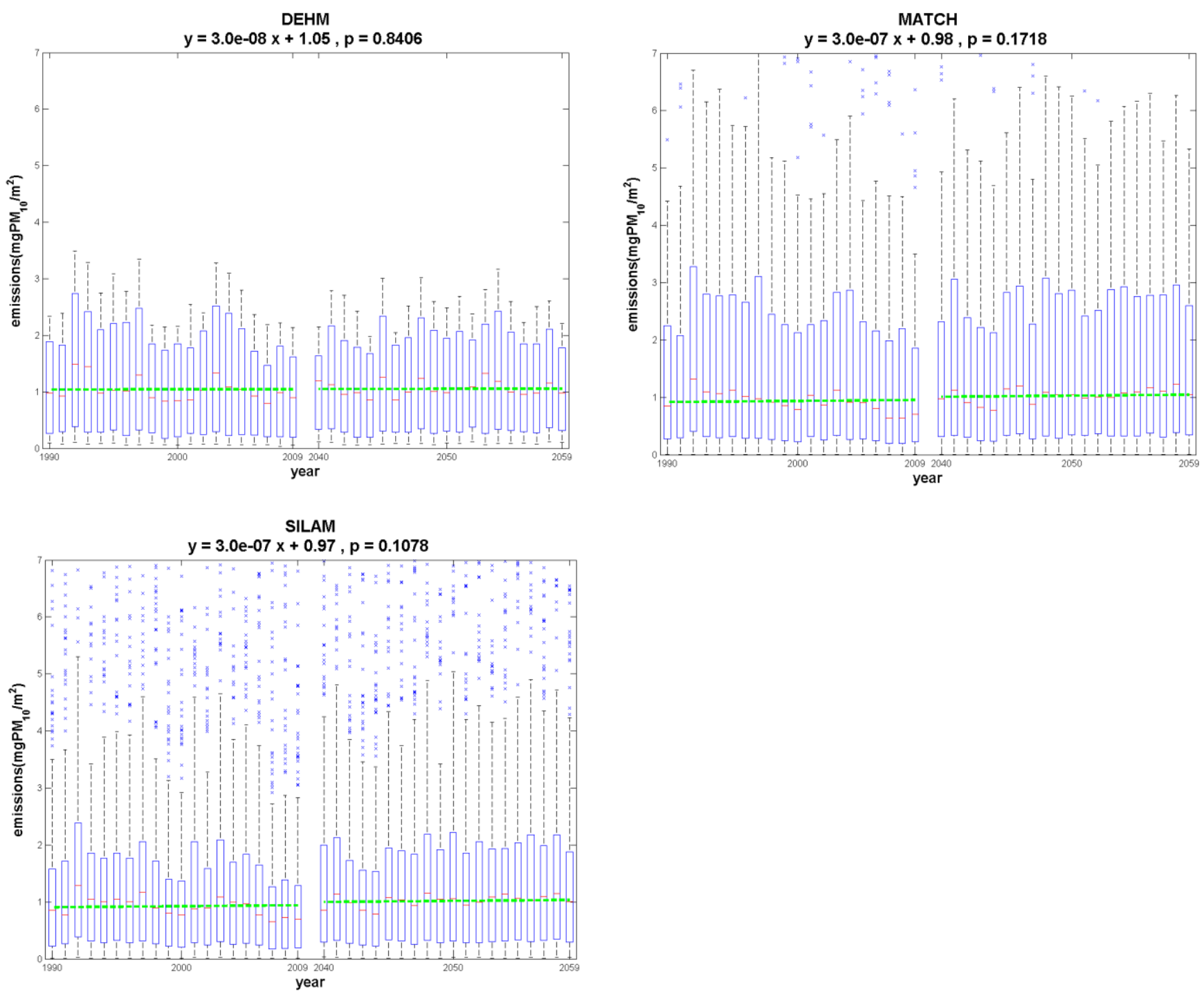

1 Figure S5 Baltic Sea SSA annual emission $\left(\mathrm{mgPM}_{10} \mathrm{~m}^{-2}\right)$ normalized trend $(\mathrm{y})$ over the past 2 and future periods (x [year]).

3 

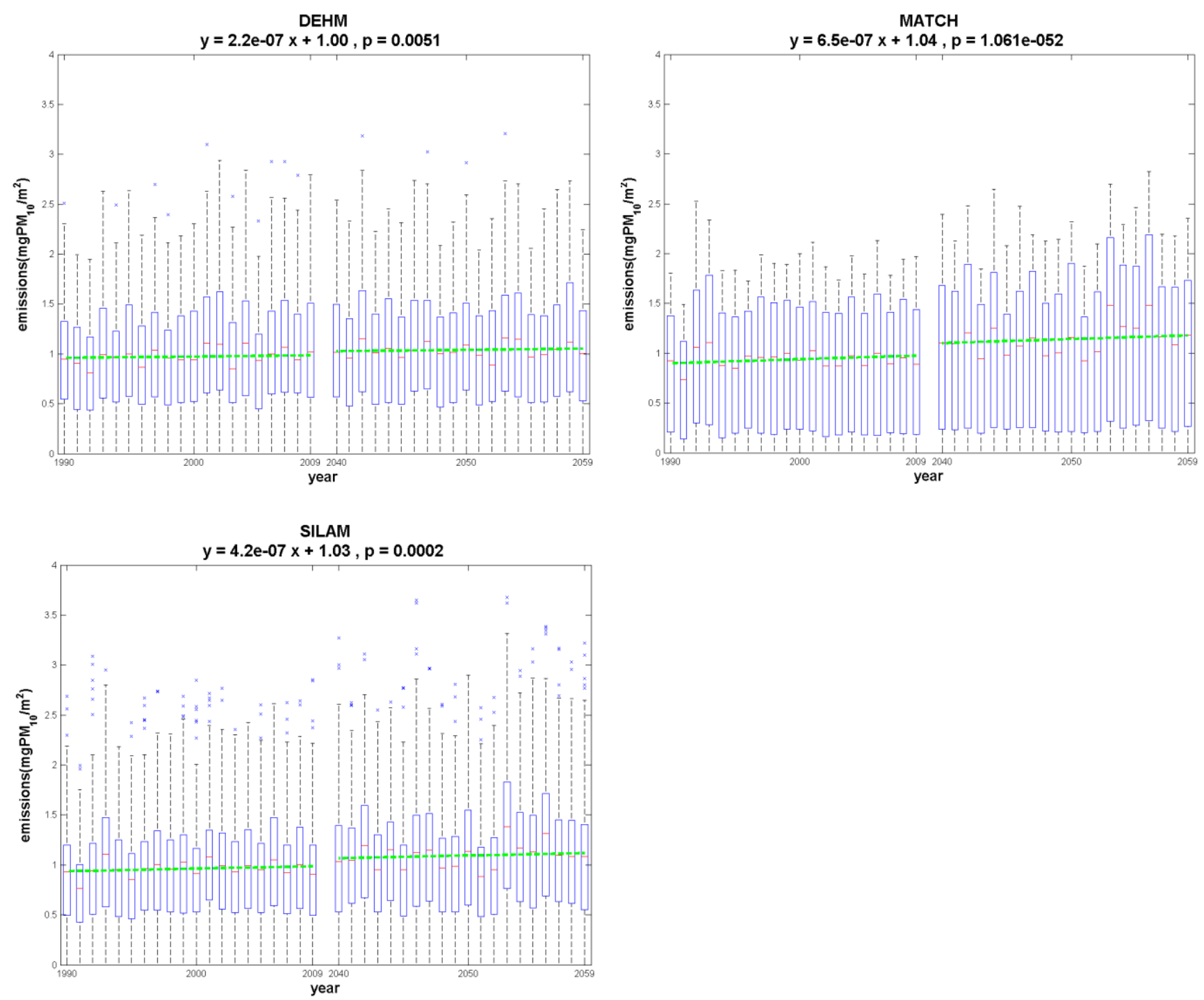

2 Figure S6 Black Sea SSA annual emission $\left(\mathrm{mgPM}_{10} \mathrm{~m}^{-2}\right)$ normalized trend $(\mathrm{y})$ over the past 3 and future periods (x [year]). 

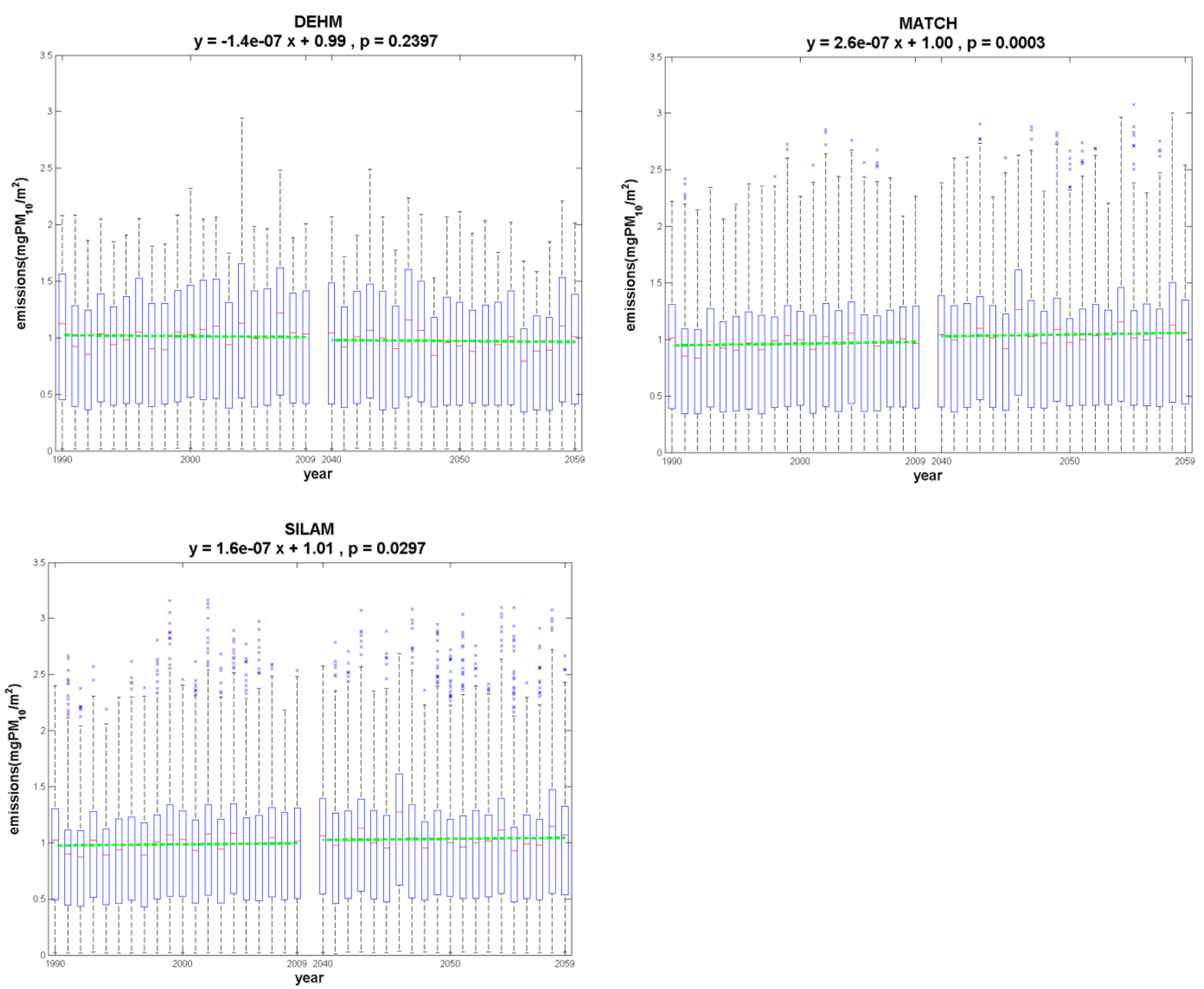

1

2 Figure S7 Mediterranean Sea SSA annual emission $\left(\mathrm{mgPM}_{10} \mathrm{~m}^{-2}\right)$ normalized trend $(\mathrm{y})$ over 3 the past and future periods (x [year]). 

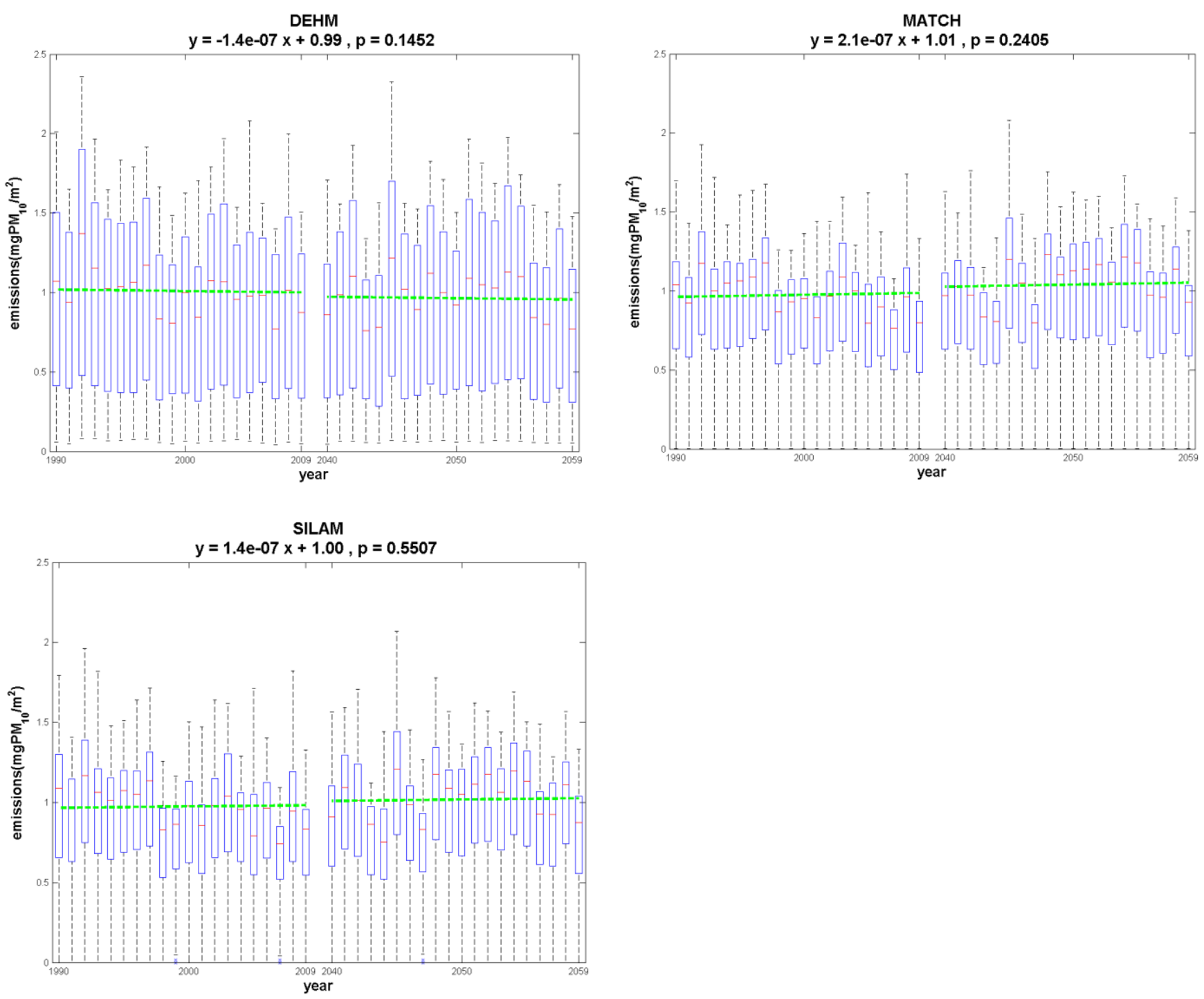

2 Figure S8 North Sea SSA annual emission $\left(\mathrm{mgPM}_{10} \mathrm{~m}^{-2}\right)$ normalized trend $(\mathrm{y})$ over the past 3 and future periods (x [year]).

4 

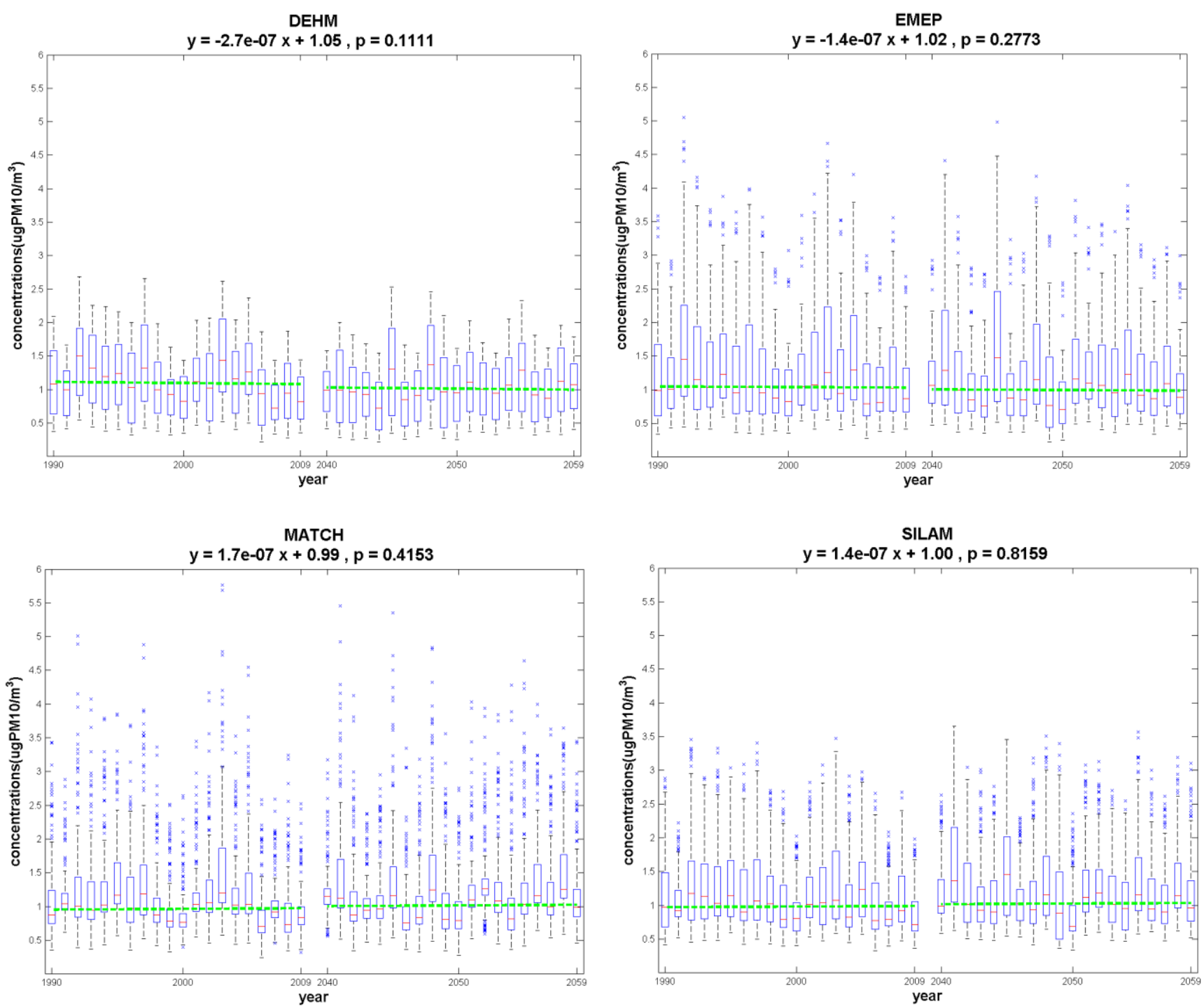

1 Figure S9 Baltic Sea SSA mean concentration $\left(\mu \mathrm{gPM}_{10} \mathrm{~m}^{-3}\right)$ normalized trend $(\mathrm{y})$ over the 2 past and future periods (x [year]).

3

4

5 

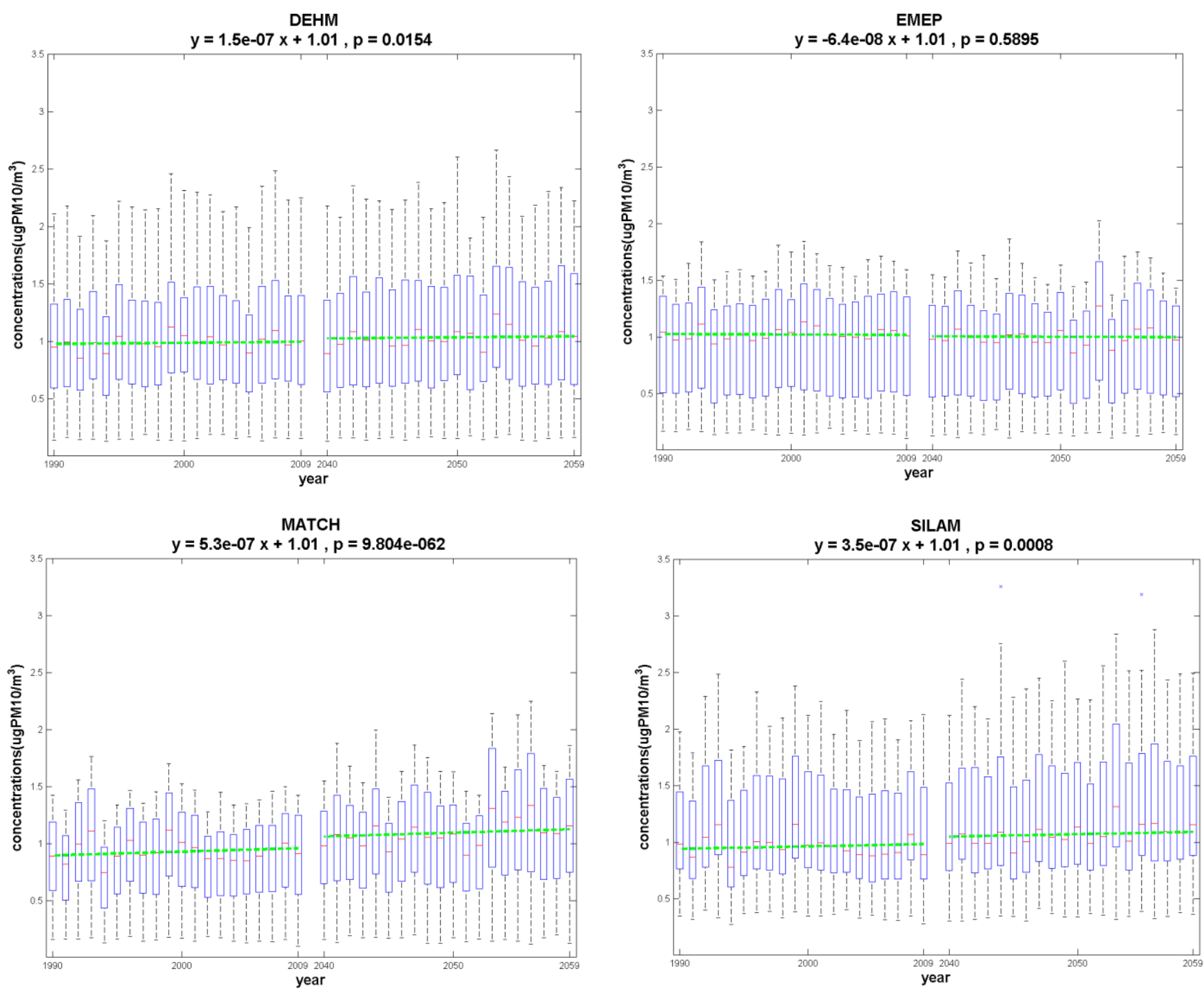

2 Figure S10 Black Sea SSA mean concentration $\left(\mu \mathrm{gPM}_{10} \mathrm{~m}^{-3}\right)$ normalized trend $(\mathrm{y})$ over the 3 past and future periods (x [year]). 

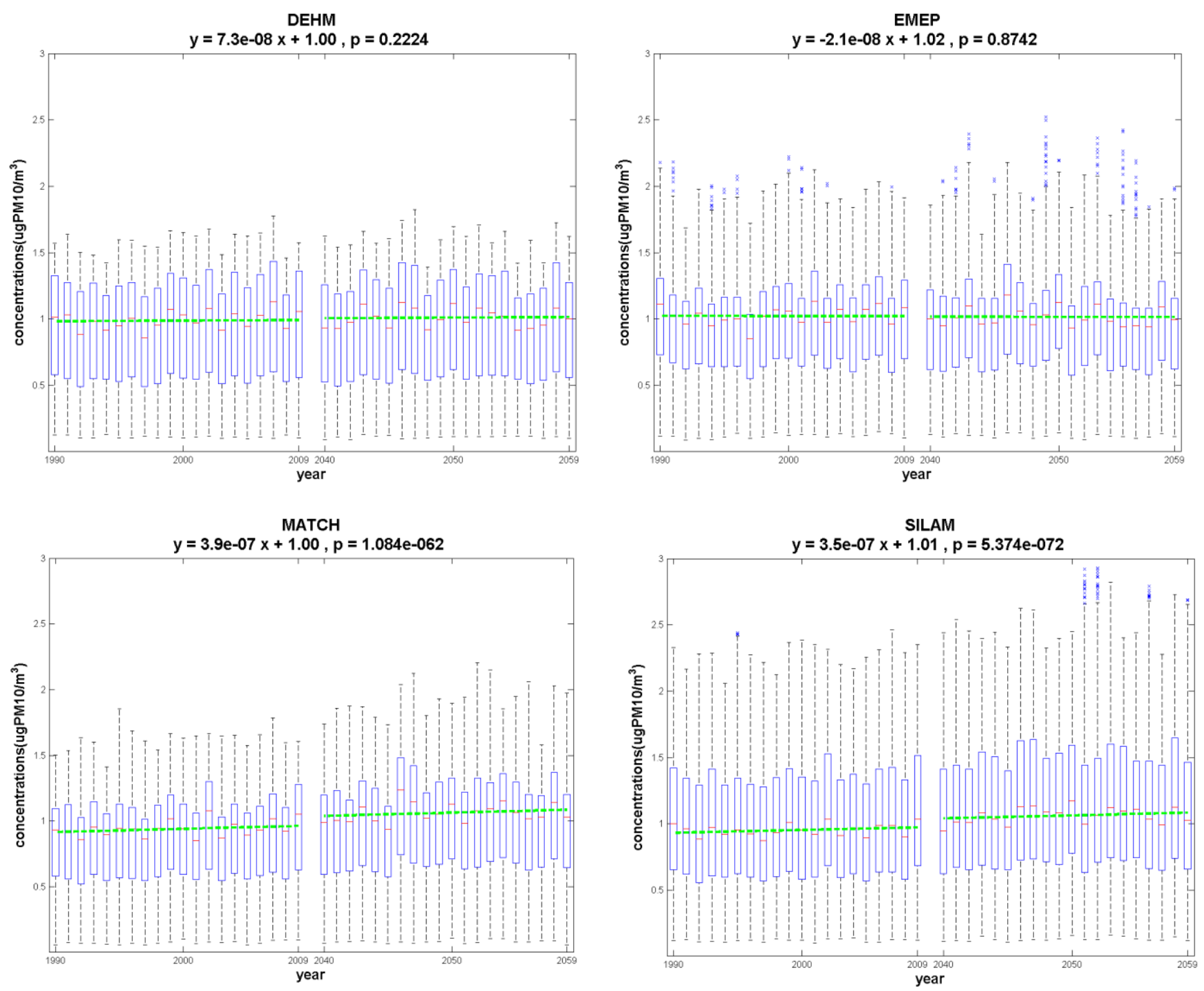

2 Figure S11 Mediterranean Sea SSA mean concentration $\left(\mu \mathrm{gPM}_{10} / \mathrm{m}^{3}\right)$ normalized trend $(\mathrm{y})$ 3 over the past and future periods (x [year]). 

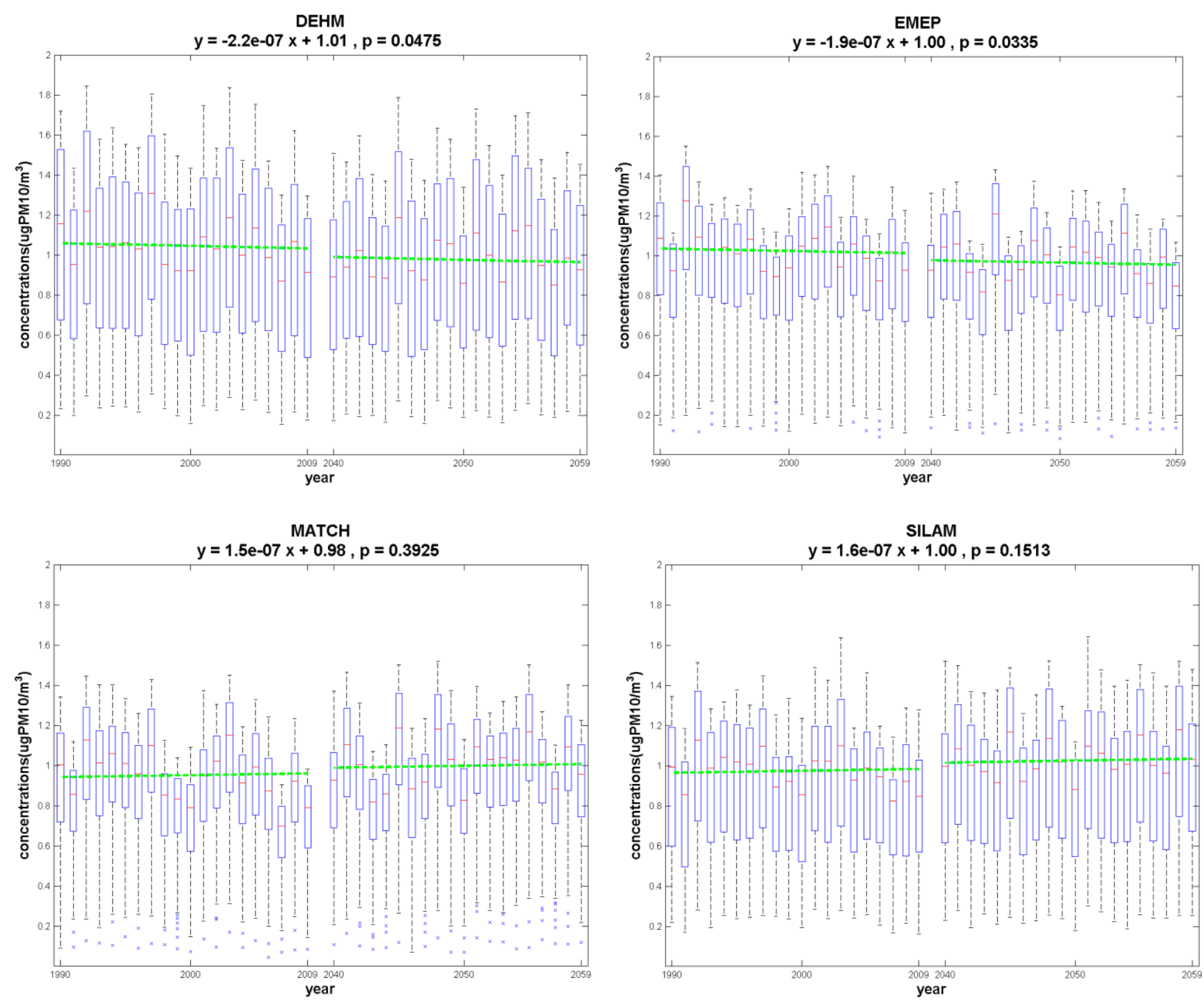

2 Figure S12 North Sea SSA mean concentration $\left(\mu \mathrm{gPM}_{10} \mathrm{~m}^{-3}\right)$ normalized trend $(\mathrm{y})$ over the 3 past and future periods (x [year]). 

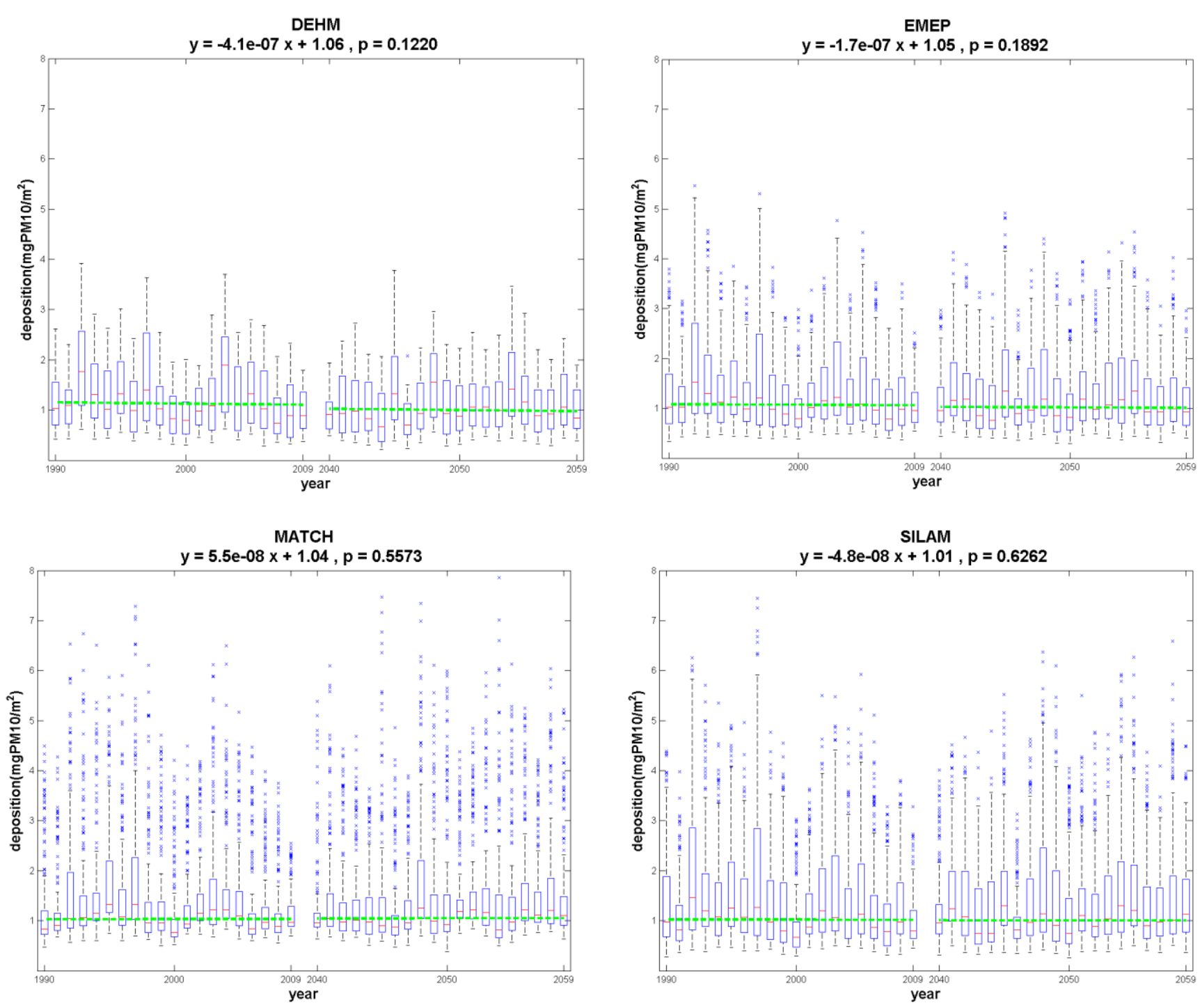

2 Figure S13 Baltic Sea SSA annual deposition $\left(\mathrm{mgPM}_{10} \mathrm{~m}^{-2}\right)$ normalized trend $(\mathrm{y})$ over the 3 past and future periods (x [year]).

4 

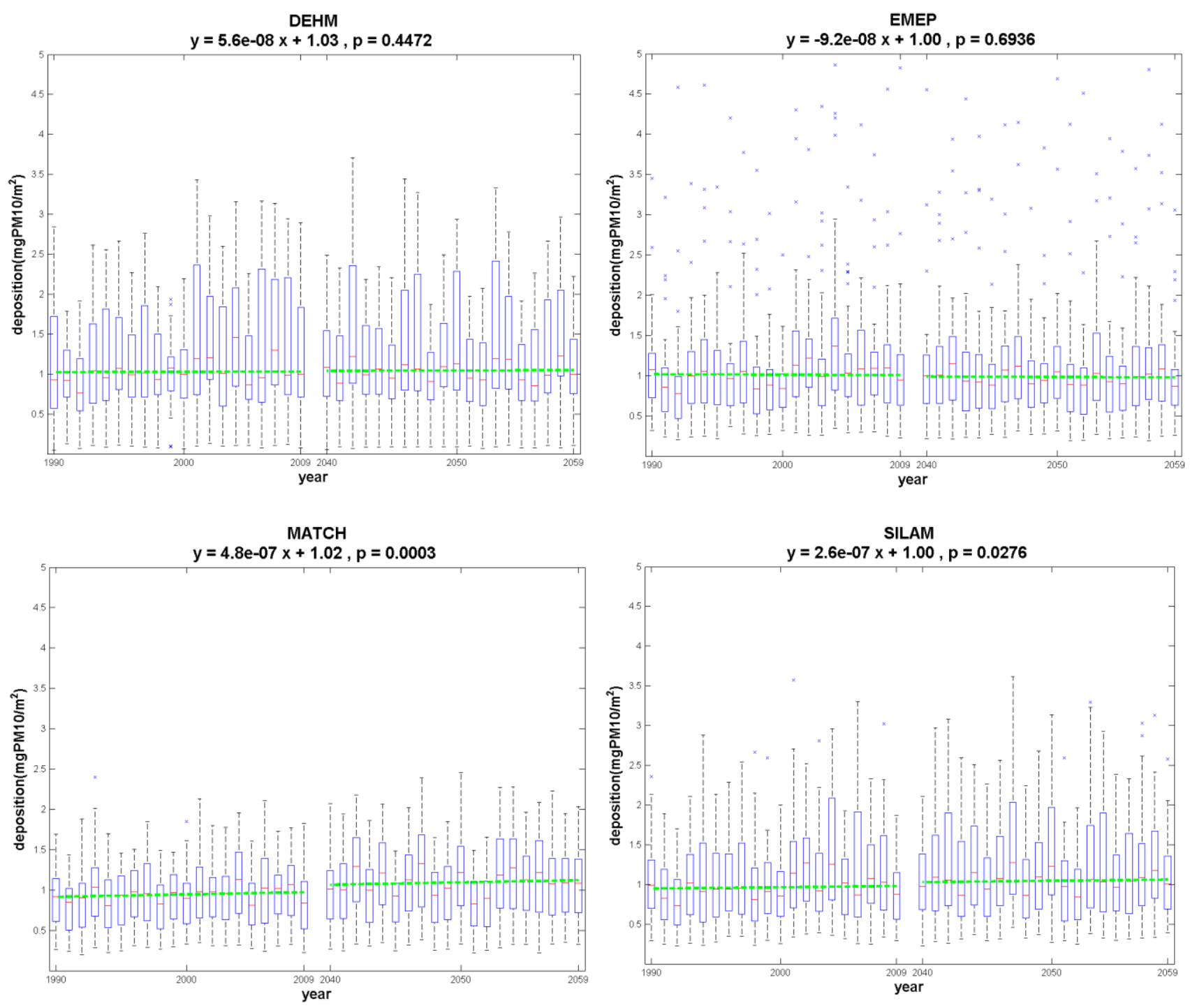

2 Figure S14 Black Sea SSA annual deposition $\left(\mathrm{mgPM}_{10} \mathrm{~m}^{-2}\right)$ normalized trend $(\mathrm{y})$ over the 3 past and future periods (x [year]). 

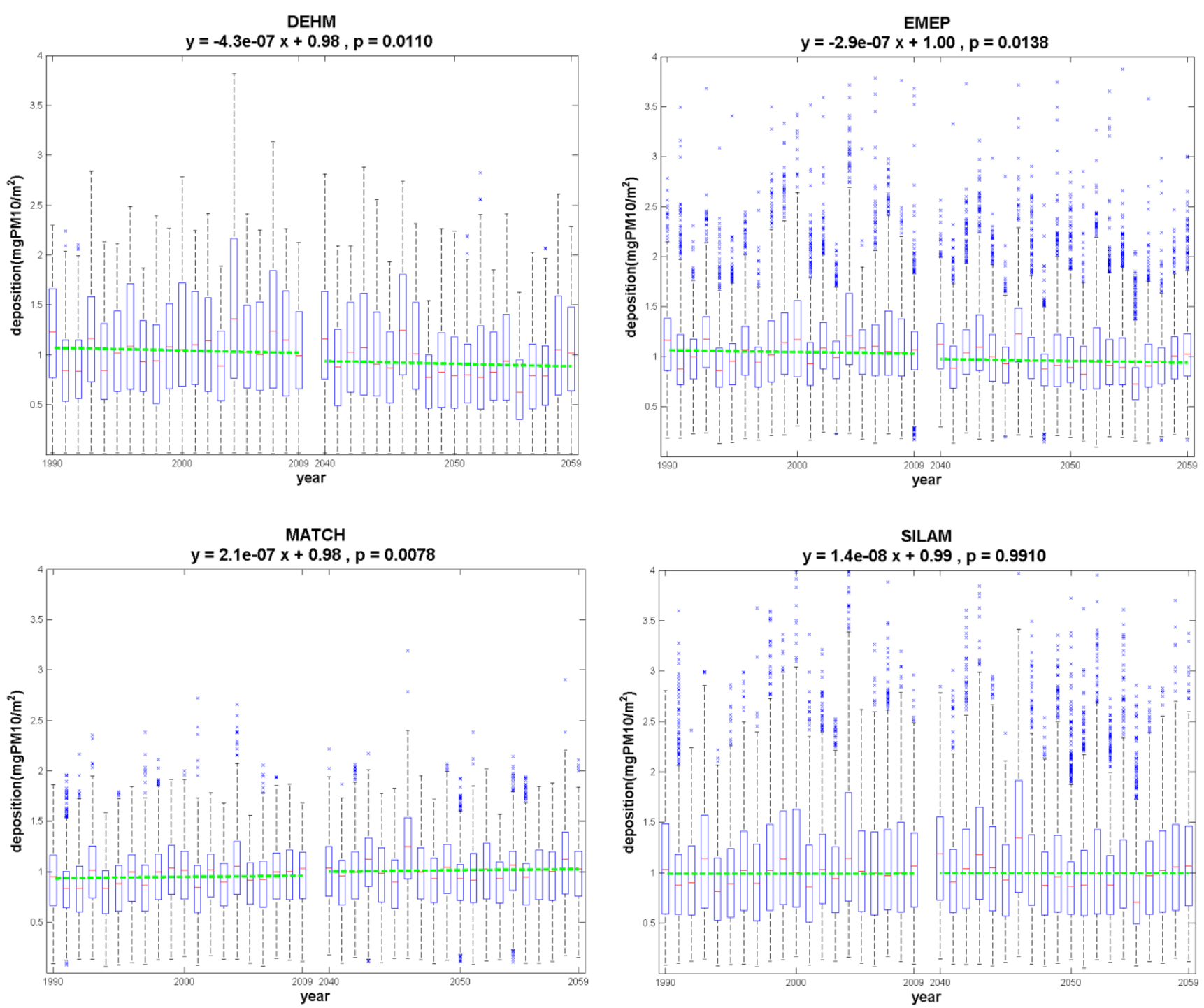

1 Figure S15 Mediterranean Sea SSA annual deposition $\left(\mathrm{mgPM}_{10} \mathrm{~m}^{-2}\right)$ normalized trend (y) 2 over the past and future periods (x [year]). 

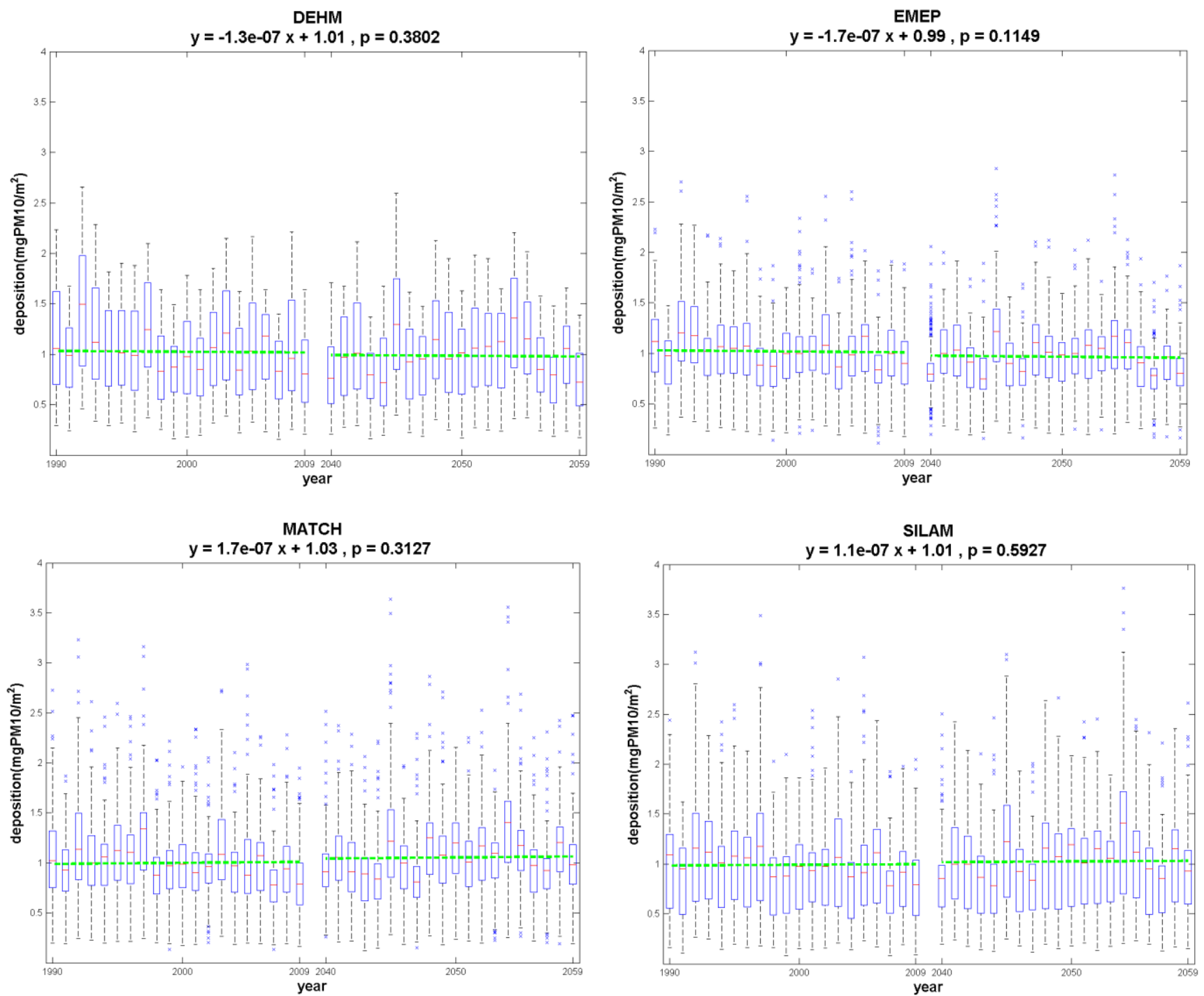

3 Figure S16 North Sea SSA annual deposition $\left(\mathrm{mgPM}_{10} \mathrm{~m}^{-2}\right)$ normalized trend $(\mathrm{y})$ over the 4 past and future periods (x [year]).

5

6

7 